\title{
Non-adherence to inhaled medications among adult asthmatic patients in Ethiopia: a systematic review and meta-analysis
}

\author{
Woldu Aberhe ${ }^{1 *}$, Abrha Hailay ${ }^{1}$, Kidane Zereabruk${ }^{1}$, Guesh Mebrahtom ${ }^{1}$ and Teklehaimanot Haile ${ }^{2}$
}

\begin{abstract}
Background: Medication non-adherence is one of a common problem in asthma management and it is the main factor for uncontrolled asthma. It can result in poor asthma control, which leads to decreased quality of life, increase hospital admission, increased health care utilization, lost productivity, and mortality. To date, there have been no studies and protocols that estimated the pooled national prevalence of non-adherence to inhaled antiasthmatic medications in Ethiopia. Therefore, the primary purpose of this systematic review and meta-analysis is to determine the pooled national prevalence of non-adherence to inhaled medications among asthmatic patients in Ethiopia.

Methods: Different database searching engines including PubMed, Scopus, Google Scholar, Africa journal online, World Health Organization afro library, and Cochrane review were systematically searched by using keywords such as "prevalence, non-adherence to inhaled medications, inhaled corticosteroids, and asthmatic patients" and their combinations. Six published observational studies that report the prevalence of non-adherence to inhaled medications were finally selected. The Preferred Reporting Items for Systematic Review and Meta-Analysis guideline was followed. Heterogeneity across the included studies was evaluated by the inconsistency index $\left(1^{2}\right)$. The randomeffect model was fitted to estimate the pooled prevalence of non-adherence to inhale anti-asthmatic medications. All statistical analysis was done using R version 3.5.3 and R Studio version 1.2.5033 software for windows.

Results: The pooled national prevalence of non-adherence to inhaled medications among asthmatic patients was $29.95 \%(95 \% \mathrm{Cl}, 19.1,40.8 \%)$. The result of this meta-analysis using the random-effects model revealed that there is high heterogeneity across the included studies. The result of subgroup analysis indicates that one out of three in the Oromia region and one out of five in the Amhara region asthmatic patients was non-adherent to their inhaled anti-asthmatic medications.
\end{abstract}

Conclusion: the prevalence of non-adherence to inhaled anti-asthmatic medications was high. Thus, our finding suggests that one out of four asthmatic patients were non-adherent to inhaled medications. The ministry of health, health policymakers, clinicians, and other health care providers should pay attention to strengthening the adherence levels to inhaled anti-asthmatic medications, and country-based interventions should be developed to reduce the burden of non-adherence to inhaled anti-asthmatic medications.

Keywords: Asthma, Ethiopia, Medications non-adherence, Meta-analysis, And prevalence

\footnotetext{
* Correspondence: aberhewoldu@gmail.com

'Department of Adult Health Nursing, School of Nursing, Aksum University, Aksum, Ethiopia

Full list of author information is available at the end of the article
}

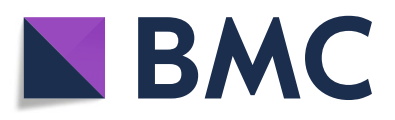

(- The Author(s). 2020 Open Access This article is licensed under a Creative Commons Attribution 4.0 International License, which permits use, sharing, adaptation, distribution and reproduction in any medium or format, as long as you give appropriate credit to the original author(s) and the source, provide a link to the Creative Commons licence, and indicate if changes were made. The images or other third party material in this article are included in the article's Creative Commons licence, unless indicated otherwise in a credit line to the material. If material is not included in the article's Creative Commons licence and your intended use is not permitted by statutory regulation or exceeds the permitted use, you will need to obtain permission directly from the copyright holder. To view a copy of this licence, visit http://creativecommons.org/licenses/by/4.0/. The Creative Commons Public Domain Dedication waiver (http://creativecommons.org/publicdomain/zero/1.0/) applies to the data made available in this article, unless otherwise stated in a credit line to the data. 


\section{Background}

Asthma is a major chronic respiratory disease that affects around 339 million people worldwide [1]. There has been an increase in the global prevalence, economic burden, morbidity, and mortality associated with asthma over the last four decades. Globally, asthma ranked 28th among the leading causes of the burden of disease and 27 th in low- and middle-income countries [1, 2]. Despite Ethiopia was signed to achieve a sustainable development goal to reduce the premature death from noncommunicable diseases by one third from 2016 to 2030 but the annual death of the Ethiopia population due to non-communicable diseases such as asthma is still high (39\%) $[3,4]$. In Ethiopia, the world health organization reported that $1.12 \%$ of the total deaths were caused by asthma and ranked 18th from the world [5].

Adherence is 'the extent to which a patient's behavior corresponds with recommendations from a health care provider' [6]. Inhaled medicines such as inhaled corticosteroids are the therapeutic use of inhaled gases that the patients breathe directly (through inhalers) into the lungs for treatment of chronic lung disease [7]. Despite the introduction of inhaled medications as the primary treatment for asthma has led to significant improvements in asthma control [1, 8], Medication non-adherence is still one of a common problem in asthma management. Adherence to asthma medication is very poor, with the prevalence of nonadherence ranging from 30 to $70 \%$. The annual economic burden of asthma in developing countries was over $\$ 20$ billion and up to three-quarters of the total costs associated with asthma are due to poor asthma control [9-13]. Nonadherence to inhaled corticosteroids is likely responsible for $24 \%$ asthma exacerbations [14]. It can result in poor asthma control, which leads to decreased quality of life, increase hospital admission, increased health care utilization, lost productivity, and mortality [15-17].

Although several studies have been published on the prevalence and associated factors of non-adherence to inhaled medications among adult asthmatic patients in Ethiopia [18-23], the general prevalence remains unknown. Therefore, the primary purpose of this systematic review and meta-analysis is to determine the pooled national prevalence of non-adherence to inhaled medications among adult asthmatic patients in Ethiopia.

\section{Objective}

This study aimed to estimate the pooled national prevalence of non-adherence to inhaled medications among adult asthmatic patients in Ethiopia.

\section{Methods}

Search strategy/ information sources

This systematic review and meta-analysis were conducted following the Preferred Reporting Items for
Systematic Reviews and Meta-Analyses (PRISMA) guidelines [24]. We searched articles reporting the prevalence of non-adherence to inhaled medications among adult asthmatic patients from (PubMed/MEDLINE, Embase, Science Direct, Scopus, Google Scholar, Web of Science, Cochran library, Africa Wide Information, World Health Organization (WHO) afro library, and Africa Index Medicus) using Electronic databases from inception to September $1 / 2020$. The following keywords were searched by using BOOLEAN (AND/OR) operators to combine search terms. "medication adherence", "inhaled corticosteroids", "non-adherence to inhaled medications", "treatment adherence," "associated factors", "asthmatic patients", "Ethiopia, "systematic review and meta-analysis", and combinations of these terms were used. The search from the above databases confirmed that there was no systematic review and /or protocol on the topic of interest. Moreover, the reference lists of eligible articles were also searched to retrieve additional relevant articles.

\section{Data extraction and quality assessment}

From each included study, information on the name of the first author, year of publication,

study area (region), health facility, study design, cases, sample size, medication adherence status, response rate, the prevalence of non-adherence to inhaled medications among adult asthmatic patients in Ethiopia were extracted using a pre-piloted template prepared in a Microsoft Excel spreadsheet. The two reviewers (WA and $\mathrm{KZ}$ ), screened the titles, abstracts of all citations retrieved, and the full-text search results to identify potentially eligible studies. When necessary, authors were contacted for additional information to confirm eligibility of studies. Where there is missing information, the corresponding author of the study was contacted to request the missing information. Emails were sent to the corresponding authors to request for additional information before excluding the study. Disagreements were resolved by discussion after mutual consensus with a third reviewer who is an experienced researcher $(\mathrm{AH})$ in meta-analysis studies. The search results were uploaded to EndNote software first to remove duplicates. Finally, 6 research papers out of 104 articles were selected.

\section{Criteria for considering studies for the review Inclusion criteria}

Design: All published and unpublished observational studies.

Population: Study participants should be at least 18 years of age.

Publication status: Only peer-reviewed articles.

Settings: Hospital-based studies.

Language: The articles included in this study were those articles published only in the English language. 
This is because of publication through other languages in Ethiopia is uncommon Publication or report year: We reviewed all publications that report the prevalence of non-adherence to inhaled medications among adult asthmatic patients in Ethiopia.

Method of diagnosis: No restriction on methods of diagnosis.

Intervention(s)/exposure(s): On inhaled anti-asthmatic medications.

Outcome: Prevalence of non-adherence to inhaled medications.

\section{Exclusion criteria}

Observational studies including case reports and case series were excluded. Studies not performed in humans, qualitative studies, studies that lack relevant data needed to compute the prevalence or frequency of nonadherence to inhaled medications, and studies among children.

\section{Quality assessment of included studies}

The methodological quality of the included studies was evaluated using the Newcastle-Ottawa Scale. The Newcastle-Ottawa Scale was designed to assess the quality of non-randomized studies in meta-analyses. This scale is primarily formulated by a star allocation system, assigning a maximum of 10 stars for the risk of bias in three areas: a selection of study groups ( 4 or 5 stars), comparability of groups ( 2 stars), and ascertainment of the outcome of interest or the exposure ( 3 stars). No validation study provides a cut-off score for rating lowquality studies; a priori, we arbitrarily established that $0-3,4-6$ and 7-10 stars would be considered at high, moderate, and low risk of bias, respectively [25].

\section{Statistical analysis and presentation of results}

Data were analyzed using the $\mathrm{R}$ version 3.5.3 and Rstudio version 1.2.5003 software. Forest plots were drawn to visualize the combined prevalence of non-adherence to inhaled medications and the extent of statistical heterogeneity between studies. Heterogeneity across the studies was assessed by Cochrane's $\mathrm{Q}$ test and $\mathrm{I}^{2}$ statistic. $\mathrm{I}^{2}$ statistic ranges from 0 to $100 \%$. $\mathrm{I}^{2}$ statistic (with values of 25,50 , and $75 \%$ is representative of a low, medium, and high heterogeneity, respectively) [26]. There was high heterogeneity between the included studies. Therefore, we used a random-effects model to estimate the overall pooled national prevalence of non-adherence to inhaled medications. A subgroup analysis was summarized by geographic regions where the study was conducted.

\section{Data management}

Based on the inclusion and exclusion criteria, a tool has been developed a priori to guide the screening and selection process. The tool was piloted and revised before data extraction begins. The search results were uploaded to EndNote software first to remove duplicates.

\section{Selection process}

Once data are obtained, two reviewers independently screen the titles and abstracts of articles retrieved from the literature search against the inclusion criteria. Full texts for the eligible titles and/or abstracts including those where there is uncertainty were obtained for further assessment on whether to include in the study or not. Where necessary, authors were contacted for additional information to confirm eligibility of studies. Disagreements were resolved through discussion and when needed there was arbitration by a third reviewer. Reasons for excluding articles were recorded.

\section{Data collection process}

Data were extracted using a standardized data extraction form. From the studies included, two assessors will independently extract data using the predefined standardized extraction form. Disagreements were resolved through discussion and when needed there was arbitration by a third reviewer.

Where there is missing information, the corresponding author of the study was contacted to request the missing information. Emails were sent to the corresponding author to request for additional information before excluding the study.

\section{Data items}

Data on general information, authors, publication year, country, and region, data collection year, study characteristics (study design, setting, case, sample size, response rate), were extracted.

\section{Outcomes and prioritization}

The primary outcome is the prevalence of nonadherence to inhaled medications among adult asthmatic patients in Ethiopia.

\section{Risk of bias in individual studies}

To assess the risk of bias and quality of studies included in this review, a tool developed by Hoy et al. for prevalence studies were used [27]. The tool contains 11 items; items 1-4 assess the external validity, 5-10 assess the internal validity, and item 11 is a summary of the overall risk by the reviewer based on the responses of the above 10 items which are scored 1 if yes and 0 if no. Studies was classified as having a low (>8), moderate [28-30], or high $(\leq 5)$ risk of bias. This exercise was done by two reviewers and disagreements were solved by discussion and where necessary by arbitration involving a third reviewer/author $(\mathrm{AH})$. 
For each included study, we will estimate the precision (C) or margin of error, considering the sample size (SS) and the observed prevalence (p) of non-adherence to inhaled medications from the formula:

$\mathrm{SS}=\frac{z^{2} * p *(1-p)}{d^{2}}$ where $Z$ was the $z$ value fixed at 1.96 across studies (corresponding to $95 \%$ confidence interval). The desirable margin of error is $5 \%(0.05)$ or lower.

\section{Data synthesis}

A meta-analysis was performed to estimate the pooled prevalence of non-adherence to inhaled medications. Results were presented using forest plots. A subgroup analysis was summarized by geographic regions where the study was conducted. A random-effects meta-analysis was performed [31] to determine the pooled estimate prevalence of non-adherence to inhaled medications in Ethiopia. Heterogeneity was explored using Cochrane's $Q$ and quantified by $I^{2}$ statistics [26]. Results were reported as proportions with corresponding 95\% confidence intervals (CIs). The results of this review were reported based on the Preferred Reporting Items for Systematic Reviews and Meta-Analyses (PRISMA) guidelines [32] (Supplementary file 1-PRISMA checklist).

\section{Results}

\section{Screening flow}

Totally 6 articles were reviewed base on the four steps of the PRISMA statement [24]. The online search process initially yielded 104 articles. Of which 30 articles duplicate records were removed. After reviewing the title and abstract, we excluded 58 irrelevant articles. From the remaining 16 articles, 10 articles were excluded since they failed to meet eligibility. Finally, a total of 6 relevant articles with 921 asthmatic patients were included in the meta-analysis. The detailed steps of the screening process are shown in a PRIS MA flow chart of the study selection (Fig. 1).

\section{Study characteristics}

In this systematic review and meta-analysis, 6 studies were included. Of the studies, $2(33.3 \%)$ were conducted in Oromia, 2(33.3\%) in Amara, and the other two were from Addis Ababa and SNNPE. All the included studies were cross-sectional studies with the sample size range from 106 to 242 asthmatic patients. The highest prevalence of non-adherence to inhaled medications was reported from SNNPE (59\%) and the lowest prevalence of non-adherence was reported in Addis Ababa (15.1\%). The quality score of each primary study, based on the
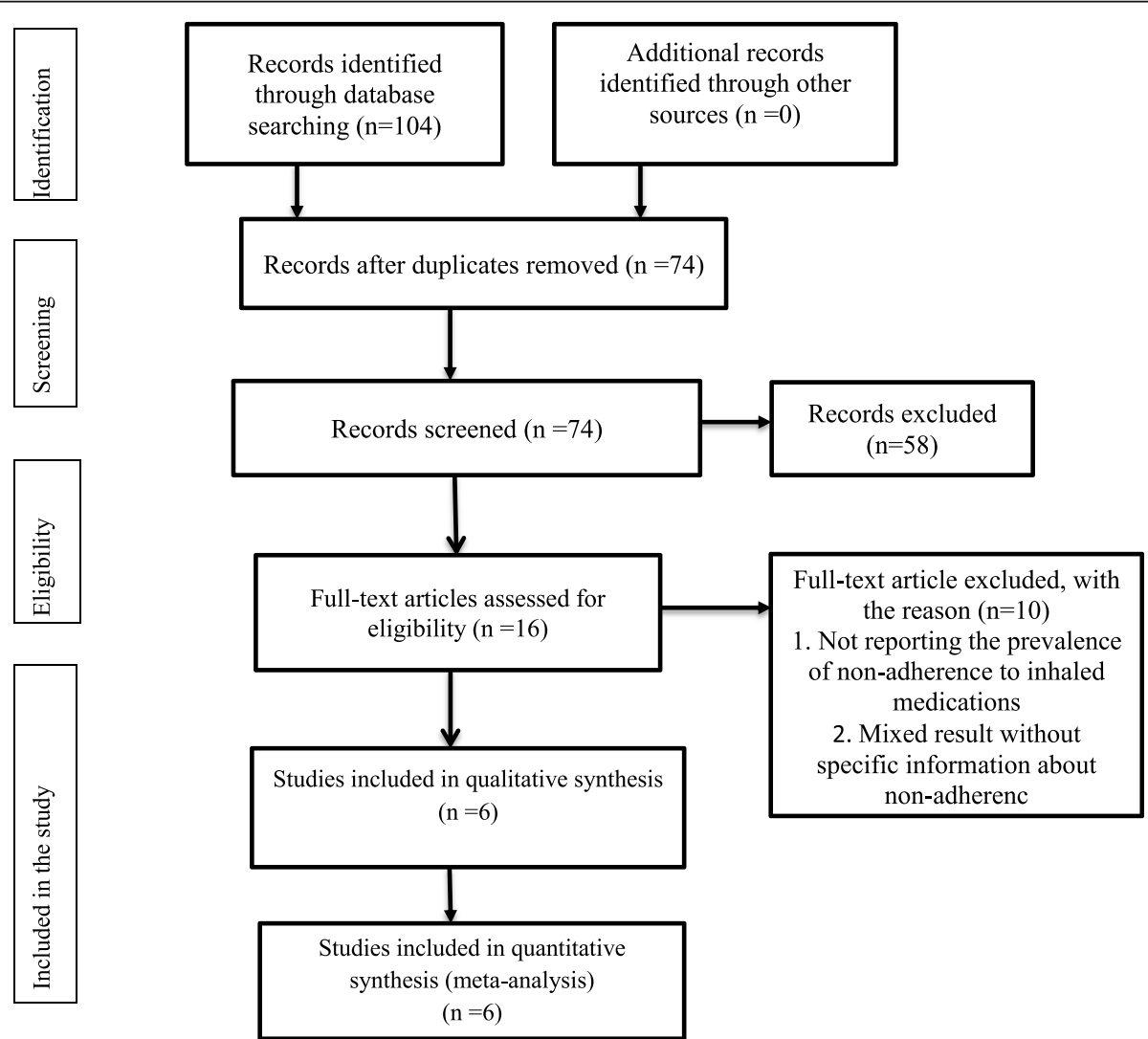

Fig. 1 Flow chart diagram for selection of articles for meta-analysis of Non-adherence to inhaled anti-asthmatic medications 
Table 1 Characteristics of studies included in the systematic review and meta-analysis of the non-adherence to inhaled medications among asthmatic patients in Ethiopia, 2020

\begin{tabular}{|c|c|c|c|c|c|c|c|c|c|c|}
\hline Authors & $\begin{array}{l}\text { publication } \\
\text { year }\end{array}$ & $\begin{array}{l}\text { data } \\
\text { collection } \\
\text { year }\end{array}$ & Region & $\begin{array}{l}\text { Health Facility } \\
\text { Name }\end{array}$ & SD & cases & $\begin{array}{l}\text { Sample } \\
\text { size }\end{array}$ & $\begin{array}{l}\text { Quality } \\
\text { assessment } \\
\text { (Based on NOS) }\end{array}$ & $\begin{array}{l}\text { response } \\
\text { rate }\end{array}$ & Prevalence $\%$ \\
\hline Ayele and Tegegn [16] & 2017 & 2016 & Amhara & hospital & CS & 30 & 164 & 9 & 100 & 18.3 \\
\hline Tesfaye et al. [17] & 2018 & 2017 & Amhara & hospital & CS & 34 & 150 & 9 & $92.60 \%$ & $22.70 \%$ \\
\hline Zewudie et al. [19] & 2019 & 2018 & Oromia & hospital & $\mathrm{CC}$ & 69 & 242 & 8 & $100 \%$ & 28.5 \\
\hline Kebede and Mamo [18] & 2019 & 2018 & Oromia & hospital & CS & 53 & 140 & 9 & $100 \%$ & 37.9 \\
\hline Koyra and Chinasho [20] & 2091 & 2018 & SNNPE & hospital & CS & 63 & 106 & 7 & $100 \%$ & 59 \\
\hline Ayele et al. [21] & 2017 & 2014 & $\mathrm{AA}$ & hospital & $\mathrm{CS}$ & 18 & 119 & 8 & $100 \%$ & 15.1 \\
\hline
\end{tabular}

AA Addis Ababa, SNNPE South Nation Nationalities and People Ethiopia, CS Cross-sectional, SD Study Design, CC case control

Newcastle Ottawa Scale quality assessment criteria, showed no considerable risk; therefore, all the studies were considered in this systematic review and metaanalysis (Table 1).

\section{The pooled prevalence of non-adherence to inhaled medications}

The pooled national prevalence of non-adherence to inhaled medications among asthmatic patients was $29.95 \%$ (95\% CI, 19.1, 40.8\%). The presence of heterogeneity was checked by $\mathrm{I}^{2}$ statistics. The result of this meta-analysis using the random-effects model revealed that there is high heterogeneity across the included studies $\left(\mathrm{I}^{2}=93 \%\right.$, $P<0.01$ ) (Fig. 2).

\section{Subgroup analysis of non-adherence to inhaled medications by region of the country}

Based on a subgroup analysis the prevalence of nonadherence to inhaled medications among asthmatic patients in the Amhara region was 20.21\% (with 95\% CI; 15.77 to $24.64 \%$ ). The result of this sub group analysis revealed that there is no heterogeneity across the included studies $\left(\mathrm{I}^{2}=0 \%, P=0.34\right)$ (Fig. 3 ).

In this subgroup analysis the prevalence of nonadherence to inhaled medications among asthmatic patients in Oromia region was 32.7\% (95\% CI; 23.6, 41.85 (Fig. 4).

\section{Discussion}

This meta-analysis was performed to produce pooled estimates of national-wide results of non-adherence to inhaled medications among asthmatic patients. The problem of non-adherence to treatment is the main reason for insufficient asthma control; maximize unwanted drug effects, disease progression, and health care costs. In this study, the pooled national prevalence of nonadherence to inhaled medications was estimated to be 29.95\% (95\% CI, 19.1, 40.8\%). The result of this systemic review and meta-analysis indicated that one out of four asthmatic patients were non-adherent to inhaled medications.

Among the included studies the prevalence of nonadherence to inhaled medications varied from $15.1 \%$ reported by Ayele et al. [23] to 59\% reported by Koyra and

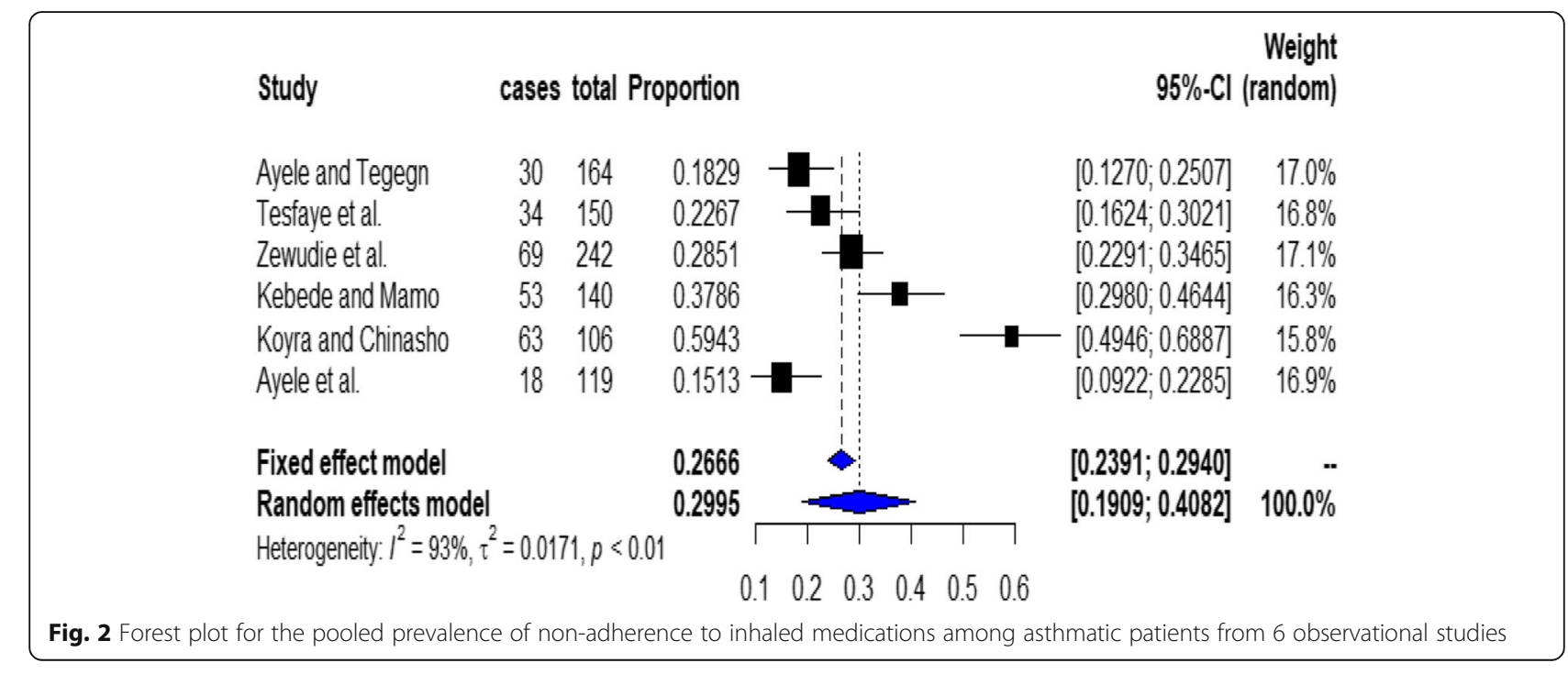




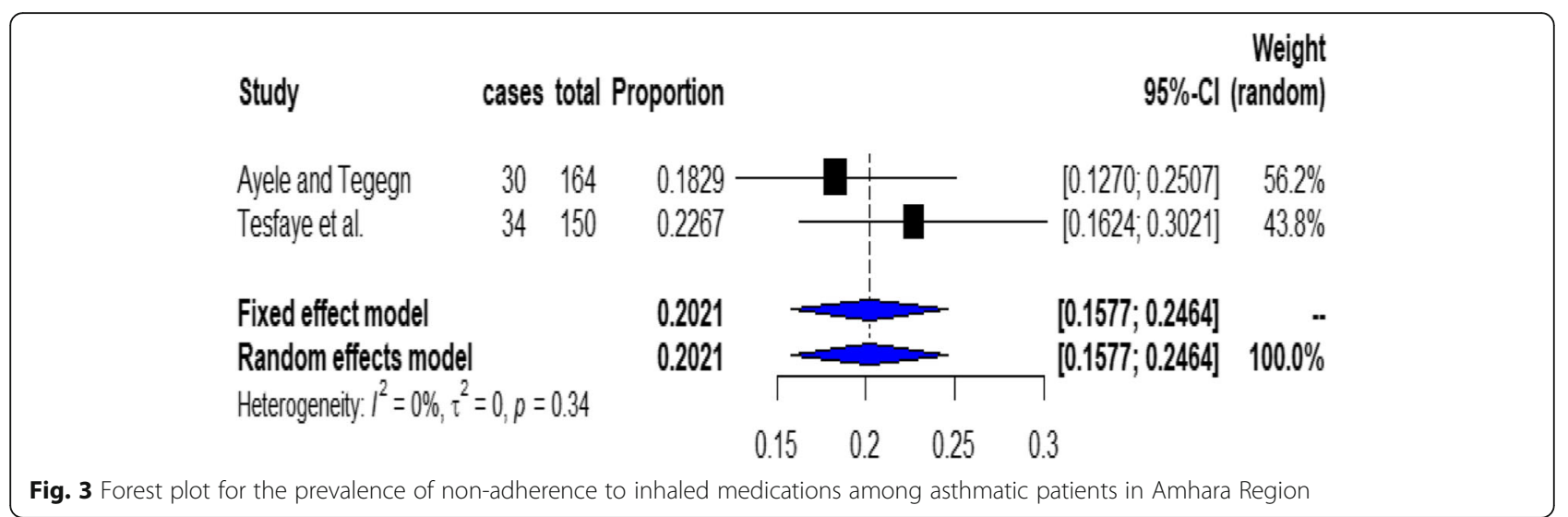

Chinasho [22]. To look at the heterogeneity of the included studies, we conducted subgroup analysis by regions. Based on subgroup analysis, the prevalence of non-adherence to inhaled medications among asthmatic patients in the Oromia (32.7\%) was higher than in the Amhara region (20.2\%). This indicates that one out of three in Oromia region and one out of five in Amhara region asthmatic patients were non-adherent to their inhaled anti-asthmatic medications.

The finding of this study was lower than the prevalence of single studies conducted in Denmark (61.3\%) [33], Northern Ireland (88\% in) [34], southeast Michigan (50\%) [35], and Kuwait (82.6\%) [36]. Whereas this finding is higher than the study conducted in Egypt (21\%) [37]. This higher or lower finding might be due to differences in sample size, socio-economic status of the population, fear of side effects, and believes on treatment ineffectiveness for controlling symptoms. Certainly, this evidence was supported by one systematic review study conducted on adherence to treatment with inhaled corticosteroids among asthmatic patients [38]. No meta-analysis study reported the prevalence of non-adherence to inhaled antiasthmatic medications in international databases. Studies reported that medication-related factors include difficulties with inhaler devices, complex regimens, side effects, cost of medication, dislike of medication, and distant pharmacies were factors are associated with nonadherence to asthma therapy $[9,11,14,39]$.

\section{Limitations of this study}

This is the first systematic review and meta-analysis study drawing the pooled national prevalence of nonadherence to inhaled medications among asthmatic patients. However, the limitation of this study was the failure to identify factors associated with non-adherence to inhaled anti-asthmatic medications. This is due to all most all of the included studies failed to report their results in a cross-tabulation form.

\section{Conclusion}

the prevalence of non-adherence to inhaled antiasthmatic medications was high. Thus, our finding suggests that one out of four asthmatic patients were nonadherent to inhaled medications.

\section{Relevance for the clinical practice}

This systematic review and meta-analysis informs health care providers', ministry of health, and health

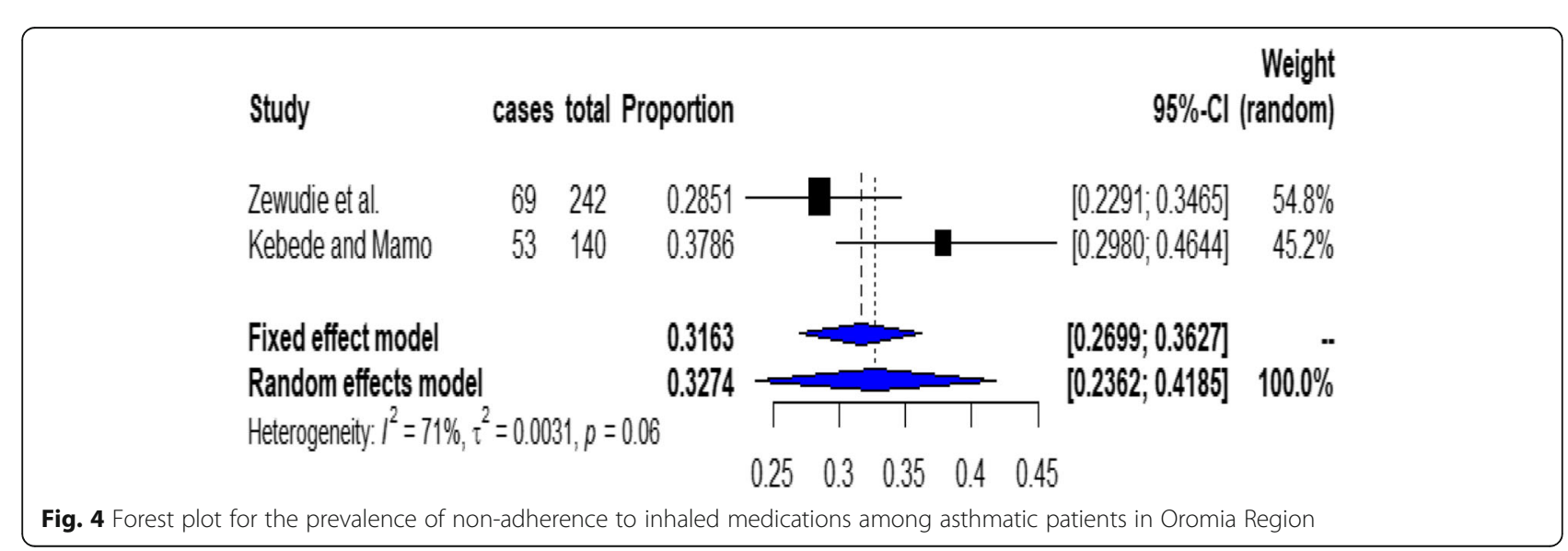


policymakers about the prevalence of non-adherence to inhaled anti-asthmatic medications. This high burden has negative effects to asthma management and outcomes. Therefore, ministry of health, health policymakers, clinicians, and other health care providers should pay attention to strengthening the adherence levels to inhaled anti-asthmatic medications and country-based interventions should be developed to reduce the burden of non-adherence to inhaled antiasthmatic medications. Reducing this prevalence will have a great contribution on asthma control; increase the quality of life, decrease hospital admission, and mortality of asthmatic patients.

\section{Supplementary information}

Supplementary information accompanies this paper at https://doi.org/10. 1186/s40733-020-00065-7.

\section{Additional file 1}

\section{Abbreviations}

Cl: Confidence Interval; PRISMA: Preferred Reporting Items for Systematic Reviews and Meta-Analyses; SNNPE: Southern Nations Nationalities, and Peoples of Ethiopia; Nations: Nationalities, and Peoples, and Ethiopia

\section{Acknowledgments}

Not applicable.

\section{Authors' contributions}

WA, AH, GM, KZ, and TH developed the protocol and involved in the design, selection of study, data extraction, and statistical analysis and developing the initial drafts of the manuscript. AH, GM, and $\mathrm{KZ}$ and involved in data extraction, quality assessment, statistical analysis, and revising. WA, TH, AH, and KZ prepared the final draft of the manuscript. All authors read and approved the final draft of the manuscript.

\section{Funding}

Not applicable.

\section{Availability of data and materials}

The data analyzed during the current meta-analysis is available from the corresponding author on a reasonable request.

\section{Ethics approval and consent to participate}

Not applicable.

\section{Consent for publication}

Not applicable.

\section{Competing interests}

The authors declare that they have no competing interests.

\section{Author details}

'Department of Adult Health Nursing, School of Nursing, Aksum University, Aksum, Ethiopia. ${ }^{2}$ Department of Maternity and Neonatal Nursing, School of Nursing, Aksum University, Aksum, Ethiopia.

Received: 23 September 2020 Accepted: 5 October 2020

Published online: 14 October 2020

\section{References}

1. Network BTSSIG. British guideline on the management of asthma. Thorax. 2008;63:iv1.

2. Vos T, Barber RM, Bell B, Bertozzi-Villa A, Biryukov S, Bolliger I, et al. Global, regional, and national incidence, prevalence, and years lived with disability for 301 acute and chronic diseases and injuries in 188 countries, 1990-2013: a systematic analysis for the global burden of disease study 2013. Lancet. 2015;386(9995):743-800

3. Shiferaw F, Letebo M, Misganaw A, Feleke Y, Gelibo T, Getachew T, et al. Non-communicable Diseases in Ethiopia: Disease burden, gaps in health care delivery and strategic directions. Ethiop J Health Dev. 2018;32(3).

4. Assembly G. Sustainable development goals. SDGs, Transforming our world: the. 2015;2030:338-50

5. LeDuc T. World health rankings live longer live better. USA: LeDuc Media Recuperado de: http://www leducmedia com 2018.

6. Jeong YY. Adherence: implications for asthma treatment. Allergy Asthma Respi Dis. 2013;1(2):105-10.

7. Kelloway JS, Wyatt RA, Adlis SA. Comparison of patients' compliance with prescribed oral and inhaled asthma medications. Arch Intern Med. 1994; 154(12):1349-52.

8. Bateman ED, Hurd S, Barnes P, Bousquet J, Drazen J, FitzGerald M, et al. Global strategy for asthma management and prevention: GINA executive summary. Eur Respir J. 2008;31(1):143-78.

9. Bender BG, Bender SE. Patient-identified barriers to asthma treatment adherence: responses to interviews, focus groups, and questionnaires. Immunol Allergy Clin. 2005;25(1):107-30.

10. Boulet L-P, Vervloet D, Magar Y, Foster JM. Adherence: the goal to control asthma. Clin Chest Med. 2012;33(3):405-17.

11. National Heart L, Institute B. Global strategy for asthma management and prevention. http://www ginasthma com/. 2002.

12. Nurmagambetov T, Kuwahara R, Garbe P. The economic burden of asthma in the United States, 2008-2013. Ann Am Thoracic Soc. 2018;15(3):348-56.

13. Ait-Khaled N, Enarson DA, Bissell K, Billo N. Access to inhaled corticosteroids is key to improving quality of care for asthma in developing countries. Allergy. 2007;62(3):230-6.

14. Williams LK, Joseph CL, Peterson EL, Wells K, Wang M, Chowdhry VK, et al. Patients with asthma who do not fill their inhaled corticosteroids: a study of primary nonadherence. J Allergy Clin Immunol. 2007;120(5):1153-9.

15. Baena-Cagnani CE. The global burden of asthma and allergic diseases: the challenge for the new century. Curr Allergy Asthma Rep. 2001;1 (4):297-8.

16. Melani AS, Bonavia M, Cilenti V, Cinti C, Lodi M, Martucci P, et al. Inhaler mishandling remains common in real life and is associated with reduced disease control. Respir Med. 2011:105(6):930-8.

17. Rau JL. Determinants of patient adherence to an aerosol regimen. Respir Care. 2005:50(10):1346-59.

18. Ayele AA, Tegegn HG. Non adherence to inhalational medications and associated factors among patients with asthma in a referral hospital in Ethiopia, using validated tool TAl. Asthma Res Pract. 2017;3(1):7.

19. Tesfaye ZT, Gebreselase NT, Horsa BA. Appropriateness of chronic asthma management and medication adherence in patients visiting ambulatory clinic of Gondar University Hospital: a cross-sectional study. World Allergy Organ J. 2018;11:18.

20. Kebede B, Mamo G. Determinants of non-adherence to inhaled steroids in adult asthmatic patients on follow up in referral hospital, Ethiopia: crosssectional study. Asthma Res Pract. 2019;5(1):1-8.

21. Zewudie A, Niqussie T, Mamo Y, Kumela K. Determinants of poorly controlled asthma among asthmatic patients in Jimma University medical center, Southwest Ethiopia: a case control study. BMC Res Notes. 2019:12(1):525.

22. Koyra H, Chinasho T. Treatment adherence and factors affecting among adult Asth-matic patients at Soddo Christian general hospital, Southern Ethiopia: Cross-Sectional Study. Int J Re-spir Pulm Med. 2019;6:121.

23. Ayele Y, Engidawork E, Bayisa T. Assessment of inhaled corticosteroids use and associated factors among asthmatic patients attending Tikur Anbessa specialized hospital, Ethiopia. BMC research notes. 2017;10(1):314.

24. Kleijnen J, Moher D. The PRISMA statement for reporting systematic reviews and meta-analyses of studies that evaluate health care interventions: explanation and elaboration; 2009

25. Shea BJ, Reeves BC, Wells G, Thuku M, Hamel C, Moran J, et al. AMSTAR 2: a critical appraisal tool for systematic reviews that include randomised or non-randomised studies of healthcare interventions, or both. BMJ. 2017;358: j4008.

26. Higgins JP, Thompson SG, Deeks JJ, Altman DG. Measuring inconsistency in meta-analyses. Bmj. 2003;327(7414):557-60.

27. Hoy D, Brooks P, Woolf A, Blyth F, March L, Bain C, et al. Assessing risk of bias in prevalence studies: modification of an existing tool and evidence of interrater agreement. J Clin Epidemiol. 2012;65(9):934-9. 
28. Organization WH. Uniting against NCDs: the time to act is now. The Brazzaville declaration on non-communicable diseases prevention and control in the WHO African region. Geneva, Switzerland: World Health Organization, Regional Office for Africa; 2011.

29. Wong ND, Lopez VA, L'Italien G, Chen R, Kline SEJ, Franklin SS. Inadequate control of hypertension in US adults with cardiovascular disease comorbidities in 2003-2004. Arch Intern Med. 2007;167(22):2431-6.

30. Guthrie B, Payne K, Alderson P, McMurdo ME, Mercer SW. Adapting clinical guidelines to take account of multimorbidity. Bmj. 2012;345:e6341.

31. DerSimonian R, Laird N. Meta-analysis in clinical trials. Control Clin Trials. 1986;7(3):177-88

32. Liberati A, Altman DG, Tetzlaff J, Mulrow C, Gøtzsche PC, loannidis JP, et al. The PRISMA statement for reporting systematic reviews and meta-analyses of studies that evaluate health care interventions: explanation and elaboration. Ann Intern Med. 2009:151(4):W-65-94.

33. Nguyen TC, Madsen H, Pottegard A, Lassen A. Adherence to maintenance medication in asthma in patients admitted with acute asthma. J Lung Pulm Respir Res. 2014;1 (1):00004.

34. Gamble J, Stevenson M, McClean E, Heaney LG. The prevalence of nonadherence in difficult asthma. Am J Respir Crit Care Med. 2009;180(9): $817-22$.

35. Williams LK, Pladevall M, Xi H, Peterson EL, Joseph C, Lafata JE, et al. Relationship between adherence to inhaled corticosteroids and poor outcomes among adults with asthma. J Allergy Clin Immunol. 2004;114(6): 1288-93.

36. Albassam A, Alharbi A, Awaisu A. Assessing adherence to inhaled corticosteroids among adults with asthma in Kuwait using the medication adherence report scale for asthma. Patient Preference and Adherence. 2020; 14:963-70.

37. Rifaat $\mathrm{N}$, Abdel-Hady $\mathrm{E}$, Hasan AA. The golden factor in adherence to inhaled corticosteroid in asthma patients. Egypt J Chest Dis Tuberculosis. 2013;62(3):371-6.

38. Charles C, Ninot G, Sultan S. Patients' illness perceptions and adherence to treatment with inhaled corticosteroids in asthma. Rev Mal Respir. 2011;28(5): 626-35.

39. Dima AL, Hernandez G, Cunillera O, Ferrer M, de Bruin M. Asthma inhaler adherence determinants in adults: systematic review of observational data. Eur Respir J. 2015;45(4):994-1018.

\section{Publisher's Note}

Springer Nature remains neutral with regard to jurisdictional claims in published maps and institutional affiliations.

Ready to submit your research? Choose BMC and benefit from:

- fast, convenient online submission

- thorough peer review by experienced researchers in your field

- rapid publication on acceptance

- support for research data, including large and complex data types

- gold Open Access which fosters wider collaboration and increased citations

- maximum visibility for your research: over $100 \mathrm{M}$ website views per year

At $\mathrm{BMC}$, research is always in progress.

Learn more biomedcentral.com/submissions 